\title{
High Velocity Impact Characteristics of Shear Thickening Fluid Impregnated Kevlar Fabric
}

\author{
Yurim Park*, Abrar H. Baluch**, YunHo Kim*** and Chun-Gon Kim**** \\ Department of Aerospace Engineering, Korea Advanced Institute of Science and Technology, Daejeon 305-701,
}

\begin{abstract}
The development of high performance fabrics have advanced body armor technology and improved ballistic performance while maintaining flexibility. Utilization of the shear thickening phenomenon exhibited by Shear Thickening Fluids (STF) has allowed further enhancement without hindering flexibility of the fabric through a process of impregnation. The effect of STF impregnation on the ballistic performance of fabrics has been studied for impact velocities below $700 \mathrm{~m} / \mathrm{s}$. Studies of STF-impregnated fabrics for high velocity impacts, which would provide a transition to significantly higher velocity ranges, are lacking. This study aims to investigate the effect of STF impregnation on the high velocity impact characteristics of Kevlar fabric by effectively dispersing silica nanoparticles in a suspension, impregnating Kevlar fabrics, and performing high velocity impact experiments with projectile velocities in the range of $1 \mathrm{~km} / \mathrm{s}$ to compare the post impact characteristics between neat Kevlar and impregnated Kevlar fabrics. $100 \mathrm{~nm}$ diameter silica nanoparticles were dispersed using a homogenizer and sonicator in a solution of polyethylene glycol (PEG) and diluted with methanol for effective impregnation to Kevlar fabric, and the methanol was evaporated in a heat oven. High velocity impact of STF-impregnated Kevlar fabric revealed differences in the post impact rear formation compared to neat Kevlar.
\end{abstract}

Key words: Shear Thickening Fluid, High Velocity Impact, Energy Absorption, STF Impregnated Kevlar Fabric

\section{Introduction}

With the development of civilization, human conflict has risen throughout history and with it the development of firearms. Such weaponry has been consistently developed in order to become more effective and powerful for combat, and in conjunction, protection against such ballistic weapons has also been pursued. Initially, such protection was comprised of cumbersome, thick, and large metallic plates to physically block firearm projectiles from penetrating the human skin resulting in life-threatening harm. However, such uncomfortable plates interfere with the mobility of the bearer. Thus, lightweight, flexible, yet protective soft body armor was critically realized with the development of high performance fabric Kevlar ${ }^{\circledast}$ in 1971, providing a means to developingeffective body armor whilst not disturbing the mobility of the bearer. Development of other high performance fabrics also offered a wider range of options in the development of effective flexible body armor. Also, numerous materials have been proposed as modifiers to enhance the performance of the fabrics. The use of shear thickening fluid (STF) as an added material to high tenacity fabrics to enhance ballistic performance has received substantial attention as its impregnation into the fabric had little contribution to the thickness and stiffness of the fabric while providing the phenomenon of shear thickening which is inherent to STF. STF is a colloidal suspension of solid particles suspended in fluid and exhibits the shear thickening phenomenon which translates from a liquid-like state to a more solid-like state due to a result of hydro-clustering where the particles aggregate with greater shear rate applied, hence, resulting in increased viscosity [1]. Studies have reported and shown that STF impregnated Kevlar fabric is both thinner and more flexible than the corresponding neat Kevlar fabric with comparable ballistic performance [2]. Moreover, ultrasonic
This is an Open Access article distributed under the terms of the Creative Commons Attribution Non-Commercial License (http://creativecommons.org/licenses/by$\mathrm{nc} / 3.0 /$ which permits unrestricted non-commercial use, distribution, and reproduction in any medium, provided the original work is properly cited.

\footnotetext{
(c) * M.S. Student

** Ph. D Student

*** Ph. D Student

**** Professor, Corresponding author: cgkim@kaist.ac.kr
} 
dispersion of STF into fabric displayed superior stab resistance performance [3], implying that dispersion of the particles into the fabric was well established.

The ballistic resistance performance of Kevlar fabric impregnated with STF or colloidal suspensions has been studied actively, however, the impact velocity ranges are in the low velocity range as the major application of STF impregnated Kevlar is soft body armor development which aims to resist the impact of handheld firearm projectiles with a launch velocity mostly below $700 \mathrm{~m} / \mathrm{s}$ except for some specialized weaponry. Kim et al. investigated the ballistic energy absorption of neat and STF impregnated Kevlar fabric up to a velocity of $300 \mathrm{~m} / \mathrm{s}$, concluding that a hybrid design of flattened roll and STF impregnated Kevlar reduced the areal density by 15 20\% [4]. Lee et al. conducted ballistic tests for impact velocity at $224 \mathrm{~m} / \mathrm{s}$ on STF impregnated fabric with varying particle sizes [5]. Also, various combinations of neat and STF impregnated Kevlar fabrics were tested against ballistic impacts at $436 \mathrm{~m} / \mathrm{s}$ [6]. Similarly, Wetzel et al. studied the rheological parameter effects through ballistic experiments with impact velocities ranging from 209.1 to $266.7 \mathrm{~m} / \mathrm{s}$ [7]. In addition to ballistic resistance, stab resistance of STF impregnated Kevlar was investigated by Egres et al. with drop tower stab testing of a theoretical impact velocity ranging from 1.40 to $3.84 \mathrm{~m} / \mathrm{s}$ [8]. While most studies have focused on such low velocity ranges, hypervelocity impact testing has been conducted on STF impregnated Kevlar fabric in a limited manner such as the study by Bohannan et al., however, the STF impregnated Kevlar fabric was tested inside a stuffed Whipple configuration where the projectile impact would penetrate a bumper material before reaching the fabric [9]. Thus, ballistic resistance study of STF impregnation has not been reported for high velocity ranges above approximately $700 \mathrm{~m} / \mathrm{s}$.

A variety of studies have been conducted with regard to the performance enhancement brought about by the use of STF impregnation in the ballistic range applicable to the typical muzzle velocities of firearms within approximately $700 \mathrm{~m} / \mathrm{s}$.
However, further investigation in higher velocity ranges has not been pursued or is not publicly available while modern rifles with performance cartridges exhibit muzzle velocities in the range of $1200 \mathrm{~m} / \mathrm{s}$. Furthermore, this study aims to investigate the potential applicability of STF impregnation in high velocity ranges including and above $1 \mathrm{~km} / \mathrm{s}$ in order to assess the applicability to space shielding against hazardous micrometeoroids and space debris that space structures are exposed to in Low Earth Orbit altitudes. This study aims to investigate the effect of STF impregnation on the post high velocity impact characteristics of Kevlar fabric by effectively dispersing silica nanoparticles in a suspension and then impregnating the Kevlar fabric.

\section{Materials and Methods}

\subsection{Silica Colloidal Suspension Preparation}

The silica colloidal suspension was manufactured to be a suspension of STF made with Seahostar KE-P10 silica nanoparticles manufactured by Nippon Shokubai Co., Ltd. (Osaka, Japan) and polyethylene glycol (PEG) mixed with methanol manufactured by OCI Co., Ltd. (Seoul, Korea) in order to facilitate the efficient dispersion of the nano silica particles among the Kevlar fabric fibers. PEG was used as the solvent of the STF due to its thermal stability and low volatility, and methanol was used in the suspension to dilute the STF for enhanced dispersion of the particles and also because methanol can be conveniently evaporated at a later time once dispersion is complete.

The nano silica particles and PEG were mixed in a 65:35 weight fraction and then methanol was added to the mixture in the weight ratio of 100:70 for mixture to methanol. To enhance the mixture, the Drive DI 25 Basic homogenizer manufactured by IKA-Werke GmbH \& Co. KG (Staufen, Germany) was used for $20 \mathrm{~min}$ at 8,000 rpm and the sonicator Ultrasonic Cleaner Branson 2510 EDTH manufactured by Branson Ultrasonics Corp. (Danbury, USA) was used

Table 1. Properties of Kevlar KM2 [11]

\begin{tabular}{ccc}
\hline & Property & Values \\
\hline & Linear Density (dtex) & 667 \\
Mechanical properties of Kevlar & Tenacity (cN/tex) & 247.1 \\
KM2 yarn & Breaking Strength (N) & 165 \\
& Elongation at Break (\%) & 3.8 \\
& Modulus (cN/tex) & 5560 \\
& Moisture Regain $(\%)$ & 7 \\
\hline \multirow{3}{*}{ Specification of Kevlar KM2 } & Yarn Count (yarn/inch) & $28 \times 28$ \\
fabric & Areal Density $\left(\mathrm{g} / \mathrm{m}^{2}\right)$ & 145 \\
& Thickness $(\mathrm{mm})$ & 0.2 \\
& Fabric System & Plain Weave \\
\hline
\end{tabular}


for $20 \mathrm{~min}$ at $40 \mathrm{kHz}$ and $50^{\circ} \mathrm{C}$ bath temperature. Previous studies have shown that sonication is an effective method of dispersing the nanoparticles to fabricate STF for immersion and impregnation into Kevlar fabric including the use of homogenization and sonication to improve the dispersibility and result in dramatic improvement in puncture resistance under high speed loading conditions [10].

\subsection{Kevlar Fabric Impregnation}

The Kevlar fabric targets were prepared in dimensions of $120 \times 120 \mathrm{~mm}$. The material properties of the Kevlar fabric are summarized in Table 1. Kevlar KM2 was used as the target fabric material as it is widely employed in ballistic applications and extensive studies have been conducted on the material in this regard.

In order to compare the impact characteristics of neat Kevlar fabric targets and STF impregnated Kevlar fabrics, the weights before and after impregnation were measured to identify the STF added weight and the areal density of the target specimens so that the areal densities of the targets can be made as close as possible to each other and be the basis of comparison. When taking into consideration the aim and application background of such impact resistant material systems, the areal density is an important factor in evaluating the performance of the system as obtaining the greatest performance per areal density can be used as a comparison reference unit for various systems Thus, by having the areal density equal to each other, the specimens' post impact characteristics can be compared on equal basis. High velocity impacts above $1 \mathrm{~km} / \mathrm{s}$ and reaching into the hypervelocity range are not common on ground but are a serious threat in the space environment such as Low Earth
Orbit. Therefore, based on a space application perspective, the comparisons are conducted with equal areal density as payload mass is a critical factor that determines the cost of space launch operations.

Using the silica colloidal suspension prepared in advance, the Kevlar targets were immersed into the suspension for 1 hour for complete impregnation. Afterwards, excess fluid was removed by applying pressure to the impregnated fabric targets using a roller, and then each target was placed in a dry oven at $80^{\circ} \mathrm{C}$ until the mass matched the total mass of only the Kevlar fabric target, silica nanoparticles, and polyethylene glycol in order to evaporate the methanol in the suspension. Figure 1 shows the major steps in fabricating the colloidal suspension and immersion of the fabric.

Once the evaporation was complete, the targets with STF impregnation exhibited a slight white coloration on the surface which was the impregnated STF on the surface. 2 neat Kevlar targets and 2 STF impregnated Kevlar targets were made and their specifications are shown in Table 2. 12 STF impregnated Kevlar layers were used in order to make the 2 STF impregnated Kevlar samples and the average STF add-on weight percent was $47.57 \%$.

\subsection{Experiment}

In order to reproduce the high velocity impacts, a 2-stage light gas gun was employed. The gas gun used in this study launched projectiles in the $1 \mathrm{~km} / \mathrm{s}$ range which is greater than the ballistic range used in the Kevlar ballistic resistance performance studies to observe the effect of STF impregnation on the impact characteristics. The 2-stage light gas gun employs 2 stages to effectively pressurize helium and consequently launch the projectile, a $5.56 \mathrm{~mm}$ diameter

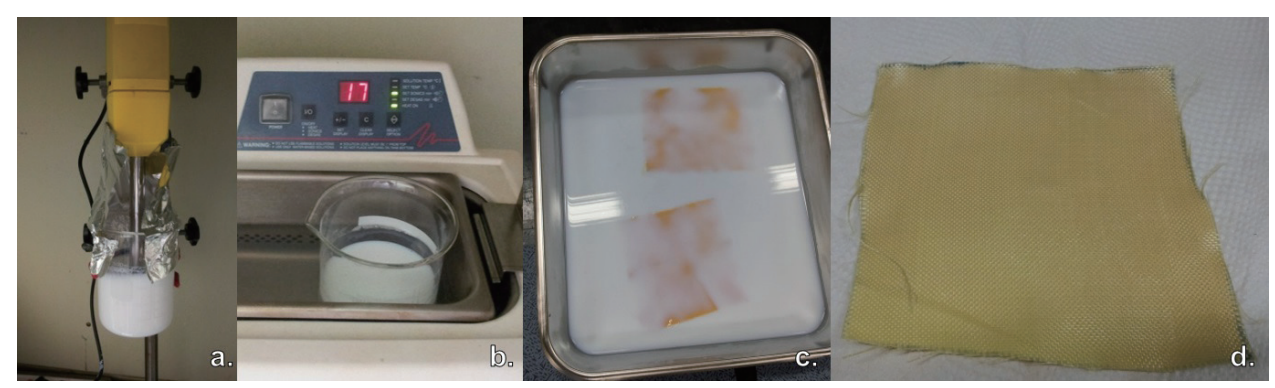

Fig. 1. Suspension preparation: (a) homogenization, (b) sonication, (c) immersion and (d) application of pressure to remove excess fluid

Table 2. Target sample specification

\begin{tabular}{ccc}
\hline & No. of Plies & Areal Density $\left(\mathrm{g} / \mathrm{m}^{2}\right)$ \\
\hline Neat Kevlar \#1 & 8 & 126.30 \\
Neat Kevlar \#2 & 8 & 126.46 \\
STF-Kevlar \#1 & 6 & 140.36 \\
STF-Kevlar \#2 & 6 & 141.88 \\
\hline
\end{tabular}




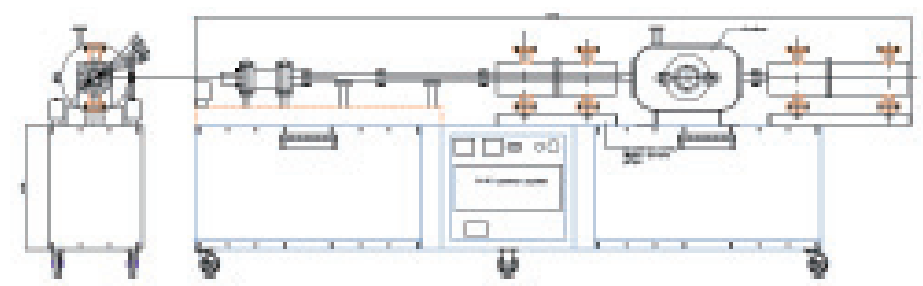

Fig. 2. 2-stage light gas gun schematic

2017-T4 aluminum sphere manufactured by SphereTec Inc. (USA). The projectile velocity before impact is measured using a commercial magnetic intervalometer which utilizes 2 magnetic coils where the passing of the conductive projectile is detected and the velocity is measured through the signal time difference between the 2 magnetic coils.

The target specimens are located and fixed within the main chamber in low vacuum condition to reproduce the space environment and remove aerodynamic resistance. Boundary conditions were limited to all 4 sides constrained completely as the basic restriction to the properties of this study. The jig that holds the specimen employed 12 screws that fix 2 metal plates together where the fabric specimen is placed between them with silicon straps grabbing all 4 sides of the specimen to establish a 4-side constrained boundary condition. The pins do not come in contact with the specimen.

Before exposing the prepared target samples to high velocity impact, the degree of dispersion of the STF on the surface of the Kevlar fabrics was investigated using SEM imaging as seen in Figure 3. The SEM images revealed that the nanoparticles were sufficiently dispersed over the surface of the yarns. The yarns were discernible and the particles were found to be relatively equally dispersed over the surface. Through visual inspection of SEM images, it was concluded that the sonication and evaporation technique produced well dispersed STF impregnated Kevlar fabrics. The samples were then remade and the prepared targets of neat Kevlar and STF impregnated Kevlar were exposed to high velocity impact inside the 2-stage light gas gun. After

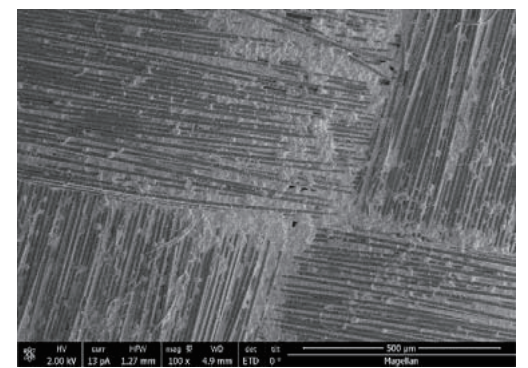

Fig. 3. SEM image of STF impregnated Kevlar fabric x100 magnification surface impact, the characteristics of the fabrics were observed in order to assess the impact characteristics of STF impregnation.

\section{Results and Discussion}

\subsection{STF Verification}

In order to verify that the fabricated STF adequately exhibited the shear thickening phenomenon which is considered the major mechanism behind the ballistic resistance enhancement provided by STF impregnation, an ARES Melt Rheometer by Rheometric Scientific was used. By increasing the shear strain applied to the STF sample, rapid increase in viscosity was observed as can be observed in Figure 4.

\subsection{Post Impact Characteristics}

After impact, the rear formation of the neat Kevlar fabric targets and the STF impregnated Kevlar fabric targets were observed and compared. Figure 5 shows the post impact characteristics of the two sample targets juxtaposed for comparison, revealing the differences that imply different energy absorption characteristics according to the impact velocity and whether STF was impregnated or not.

As evident in Fig. 5, the rear post impact formations for

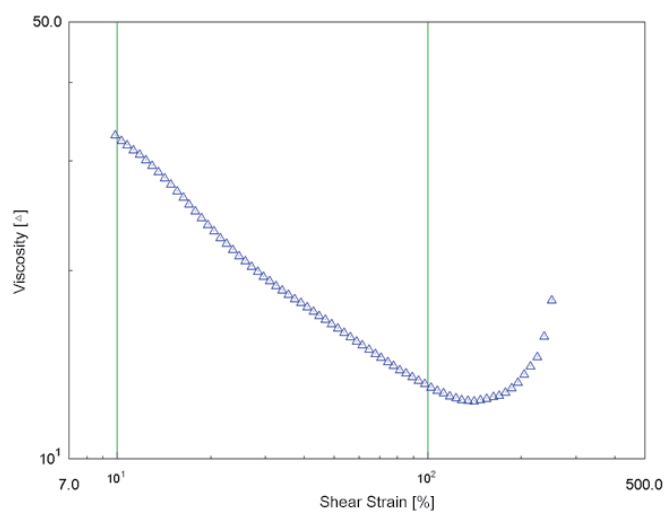

Fig. 4. Viscosity change using a parallel plate setup 
both neat Kevlar and STF impregnated Kevlar targets vary depending on the impact velocity and whether impregnation was completed or not. Due to the nature of the 2-stage light gas gun launch mechanism, the impact velocity could not be consistently matched for all targets. Thus, variations in the rear fabric shape, penetration hole size, and pulled-out fabric formation were observable.

When considering the rear fabric shape, it was observed that the most distinct pattern was exhibited for neat Kevlar with the highest impact velocity. On the other hand, the STF impregnated Kevlar targets had a less distinct pattern visible overall. Although the effect of impact velocity may be quite significant as the STF impregnated Kevlar targets did not reach the highest velocity of $1267.98 \mathrm{~m} / \mathrm{s}$, the observation that the rear bulge depths of the neat Kevlar samples were greater than that of STF impregnated Kevlar samples imply that the STF impregnation deters the maximum deformation of the fabric during and after impact. The shear thickening effect during impact may have contributed to this deterrence. Of particular note is the comparison between the $1267.98 \mathrm{~m} / \mathrm{s}$ impact velocity neat Kevlar target and the $1098.77 \mathrm{~m} / \mathrm{s}$ impact velocity STF impregnated Kevlar target. Assuming that the two impact velocities are close enough to compare the pattern assuming equal impact velocity, it can be observed that the patterns are distinctly different in that the neat Kevlar shows significant deformation in the diagonal directions expanding from the penetration hole as well as visible fabric pull along the vertical and horizontal directions to the edges while the STF impregnated Kevlar target shows little diagonal deformation.
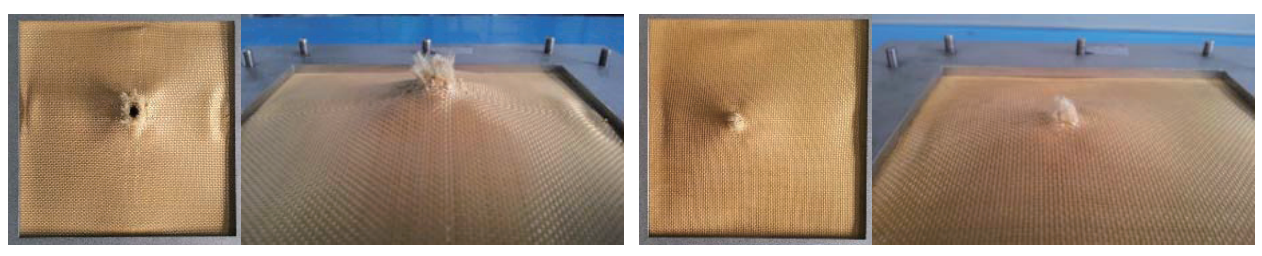

(Left) Neat Kevlar with $1267.98 \mathrm{~m} / \mathrm{s}$ impact velocity

(Right) Neat Kevlar with $865.19 \mathrm{~m} / \mathrm{s}$ impact velocity
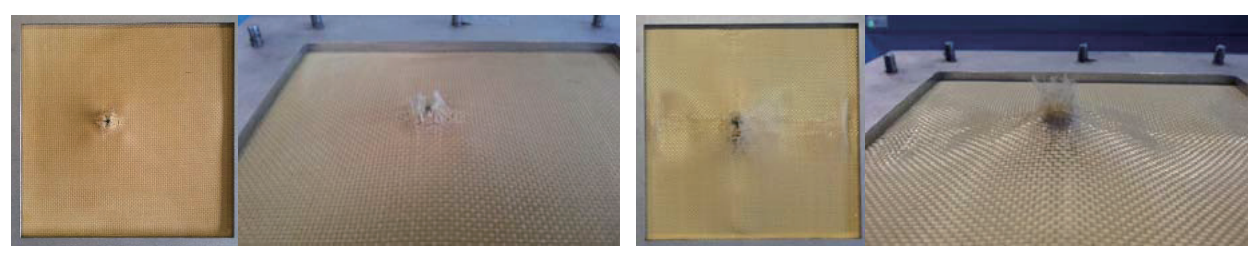

(Left) STF impregnated Kevlar with 1044.64 m/s impact velocity

(Right) STF impregnated Kevlar with 1098.77 m/s impact velocity

Fig. 5. Post impact fabric target characteristics 


\section{Conclusion}

Shear Thickening Fluid (STF) was used extensively to enhance the ballistic performance of soft body armor utilizing high tenacity fabrics such as Kevlar ${ }^{\oplus}$, however, the effect of STF impregnation on the performance of fabrics has been mostly investigated in the ballistic range below $700 \mathrm{~m} / \mathrm{s}$ only. Meanwhile, high velocity and hypervelocity impact is a critical threat for space structures in space environment and this study investigated the effect of STF impregnation in Kevlar fabrics at high velocity impacts of above $1 \mathrm{~km} / \mathrm{s}$. In this study, silica colloidal suspension composed of silica nanoparticles, polyethylene glycol, and methanol was mixed at a specific weight fraction and underwent a sonication and evaporation process to fully immerse Kevlar fabrics for STF impregnation. The degree of dispersion of STF on the surface of the Kevlar fabric was assessed through SEM imaging and was found to be relatively well dispersed about the yarns. Using a 2-stage light gas gun, the post impact characteristics of neat Kevlar targets and STF impregnated Kevlar fabric targets were observed at impact velocities in the range of approximately $1 \mathrm{~km} / \mathrm{s}$, which revealed that the STF impregnated Kevlar showed less distinct cross patterns signifying energy dissipation and greater impact resistance. In addition, it had a smaller penetration hole area with the pulled-out yarns facing inward rather than outward and the rear bulge depth was less than the neat Kevlar fabric targets. These consistent and observable characteristics imply that STF impregnation brings about a different energy absorption behavior although further study on various areal density/material combinations and effect of impact velocity on the energy absorption characteristics needs to be conducted. Extensive investigation on the effect of STF impregnation on the energy absorption mechanism of Kevlar fabric may offer STF impregnation as an effective technique to designing a shielding configuration for space applications with minimized volume and mass while maintaining or enhancing the impact resistance of the structure.

\section{Acknowledgement}

This work was supported by the National Research Foundation of Korea(NRF) grant funded by the Korea government(MEST) (No. 2012-0000133 and 2013-000378).

\section{References}

[1] Hoffman, R. L., "Explanations for the cause of shear thickening in concentrated colloidal suspensions", Journal of Rheology, Vol. 42, No. 1, 1998, pp. 111-123. DOI:10.5139/ IJASS.2010.11.4.285

[2] Lee, Y. S., Wetzel, E. D., and Wagner, N. J., “The ballistic impact characteristics of Kevlar woven fabrics impregnated with a colloidal shear thickening fluid", Journal of Materials Science, Vol. 38, Issue 13, 2003, pp. 2825-2833.

[3] Clements, F. E., and Mahfuz, H., "Enhancing the stab resistance of flexible body armor using functionalized $\mathrm{SiO} 2$ nanoparticles", $16^{\text {th }}$ International Conference on Composite Materials, Kyoto, Japan, 8-13 July 2007.

[4] Kim, C. G., Kim, I. J., Lim, G., and Yoon, B. I., “The ballistic impact characteristics of woven fabrics impregnated with a colloidal suspension and flattened rolls", Advances in Science and Technology, Vol. 71, 2010, pp. 74-79. DOI: 10.4028/www.scientific.net/AST.71.74

[5] Lee, B. W., Kim, I.-J., and Kim, C. G., "The influence of the particle size of silica on the ballistic performance of fabrics impregnated with silica colloidal suspension", Journal of Composite Materials, November 2009, Vol. 43, No. 23, pp. 2679-2698. DOI: 10.1177/0021998309345292

[6] Park, J. L., Yoon, B. I., Paik, J. G., and Kang, T. J., "Ballistic performance of $\mathrm{p}$-aramid fabrics impregnated with shear thickening fluid; Part I - Effect of laminating sequence", Textile Research Journal, April 2012, Vol. 82, No. 6, pp. 527 541. DOI: 10.1177/0040517511420753

[7] Wetzel, E. D., Lee, Y. S., Egres, R. G., Kirkwood, K. M., and Kirkwood, J. E., "The effect of rheological parameters on the ballistic properties of shear thickening fluid (STF) Kevlar composites", AIP Conference Proceedings, Vol. 712, Issue 1, 2004, pp. 288-293. DOI: 10.1063/1.1766538

[8] Egres Jr., R. G., Decker, M. J., Halbach, C. J., Lee, Y. S., Kirkwood, J. E., Kirkwood, K. M., Wagner, N. J., and Wetzel, E. D., "Stab resistance of shear thickening fluid (STF) - Kevlar composites for body armor applications", Proceedings of the $24^{\text {th }}$ Army Science Conference, Orlando, Florida, Nov. 29-Dec. 2, 2004.

[9] Bohannan, A. L., and Fahrenthold, E. P., "Simulation of STF Kevlar shielding performance in a stuffed whipple configuration", 50 ${ }^{\text {th }}$ AIAA/ASME/ASCE/AHS/ASC Structures, Structural Dynamics, and Materials Conference, Palm Springs, California, 4-7 May 2009. DOI: 10.2514/6.2009-2400

[10] Kang, T. J., Hong, K. H., and Yoo, M. R., "Preparation and properties of fumed silica/Kevlar composite fabrics for application of stab resistance material", Fibers and Polymers, Vol. 11, No. 5, 2010, pp. 719-724. DOI: 10.1007/s12221-0100719-Z

[11] Laible, R. C., Ballistic materials and penetration mechanics. Method and phenomena: their applications in science and technology, Vol. 5, Elsevier, Amsterdam, 1980, Chapter 4 . 